\title{
Perspectivas actuales de la lengua y la literatura alemanas en los estudios interculturales
}

\author{
El desafío de la interculturalidad: \\ fundamentos teóricos en literatura y comunicación ${ }^{1}$
}

VicTOr Borrero / Javier MarToS

Universidad de Sevilla

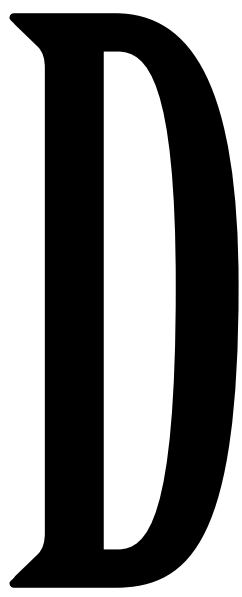

e unos años hasta ahora, el problema de la inmigración ocupa inexorablemente un lugar destacado en el horizonte de preocupaciones del ciudadano europeo. Parece que éste es el sentido de que el debate político, social y cultural se haya centrado en la última década en la definición de la naturaleza y los límites de una cultura identitaria "guía» (Leitkultur) cobrando un protagonismo creciente hasta convertirse en Alemania en palabro (Unwort) del año del año 2000. Desde su formulación por Tibi en el año 1998, la opinión pública ha asistido con asiduidad a prédicas políticas defensoras de un pacto de mínimos por la convivencia entre culturas, presidido por una lingua franca común a todas las culturas cohabitantes. La propuesta es fruto del convencimiento de que la guettización de los espacios culturales minoritarios conduce irremediablemente a la conflictividad social y al empobrecimiento cultural, como así hacen visible con severidad los resultados del informe PISA de 2003. También lo es del miedo y la incertidumbre generados por la sismología identitaria en una sociedad que ve alterarse drásticamente en un corto espacio de tiempo la representatividad de su sistema tradicional de valores, de su cultura y de su lengua.

No es de extrañar, pues, que en Alemania hayan sido los departamentos de lengua alemana para extranjeros los encargados de dar solidez disciplinar e impulso a la reflexión sobre las relaciones entre culturas en contacto, llamadas en los últimos tiempos relaciones interculturales tanto en la literatura como en la lingüística y comunicación. Las aportaciones de los filólogos Imgard Ackermann, Harald Weinrich, y Alois Wierlacher, fundador este

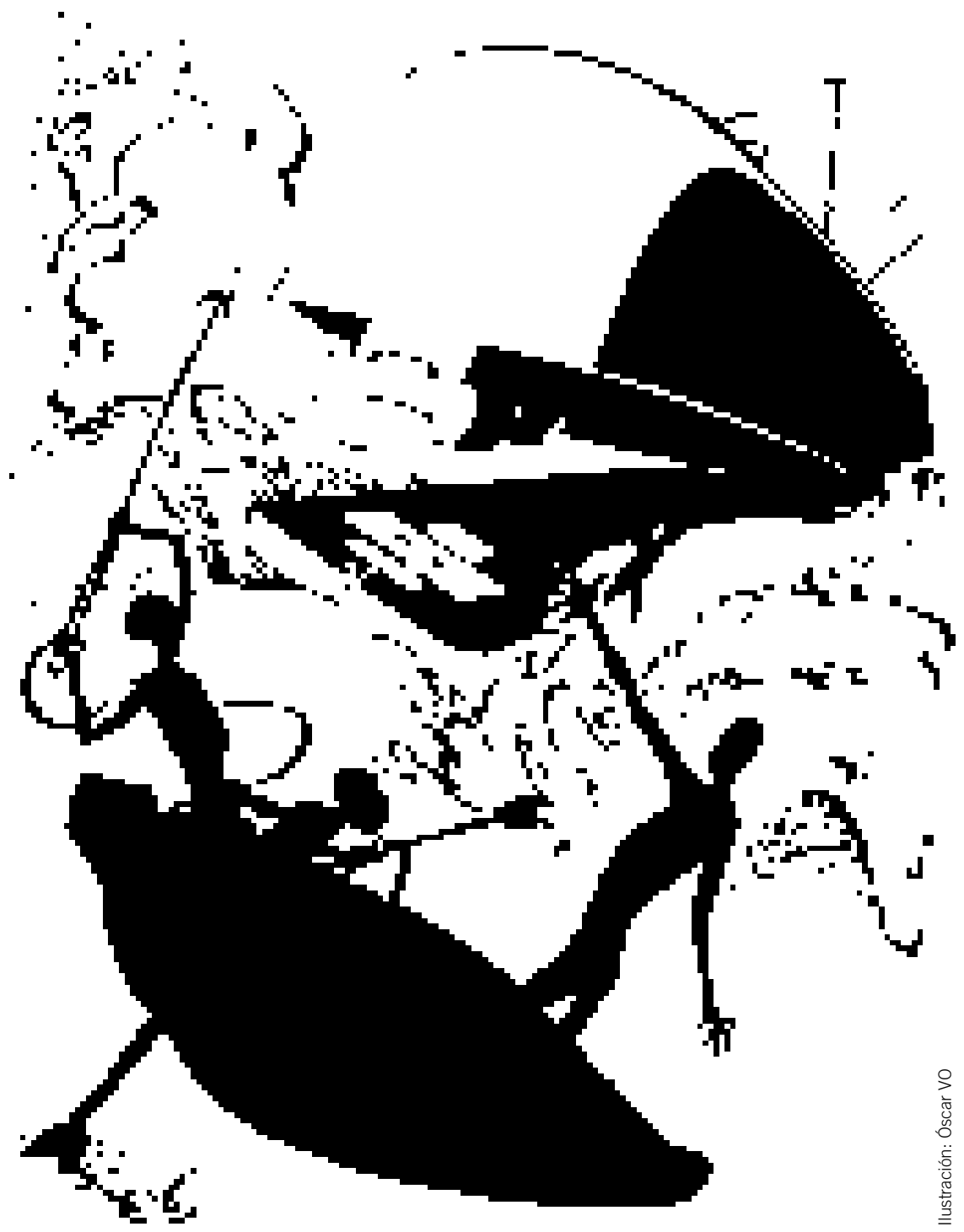

último de la llamada Germanística intercultural, cumplieron un papel primordial a través del estudio y la extensión de las metodologías docentes para la enseñanza del alemán como lengua de extranjeros. Conocedores sin duda del valor que tuviera décadas atrás el debate multicultural norteamericano en los primeros años del siglo XX y las ideas nacidas al albur del cultural pluralism de John Dewey de los años cuarenta, la escena intelectual alemana 
ralidad se aplicaría delimitando un segmento de

la producción literaria, una minoría discrimina-

da positivamente formada por un elenco de auto-

res con una aportación sociológica determinadla,

de izquierdas incorpora de este modo el término Multi-Kulti una vez es palpable en

temas, estillo y topografía de origen propios. buena parte de los aspectos de la vida que Alemania es ya, a diferencia de la Europa de los años cuarenta, un país receptor y destinatario final de la inmigración, un Einwanderungsland, pese a la negación contumaz de este hecho del lado de las autoridades gubernamentales. El ánimo de la introducción del término Multikultur no es otro, pues, que el de crear un espacio identitario plural para dar acogida a las nuevas culturas minoritarias asentadas en Alemania a partir de la oleada de inmigración invitada que, en contra de las previsiones iniciales, no retorna, de origen preponderantemente turco e italiano.

\section{Literatura intercultural}

En la particularidad del desarrollo literario, los primeros colonos del multiculturalismo ponen énfasis en la defensa de la literatura de extranjeros escrita en lengua alemana, ese espacio simbólico de convivencia, poniendo sucesivamente nombres a lo desconocido, al desconcierto, a la desorientación. En ese espacio identitario común, la lengua juega un rol capital. De ahí que el propio Weinrich denominara como Literatur der Betroffenheit, a inspiración de los autores extranjeros Franco Biondi y Rafik Shami, a aquella forma de confesión literaria que inauguraba en lengua alemana la cosmovisión del Gastarbeiter en su confrontación vivencial con los conflictos de clase y con los desarreglos y malentendidos culturales de aquella experiencia traumática. Era aquella literatura promovida por colectivos de artistas inmigrantes como «PoliKunst» o «Buchstäblich».

La protección del valor de excepcionalidad de todo lo escrito por autores foráneos se fue extendiendo en el transcurso de esos años ochenta y noventa con marbetes como Ausländerliteratur (Ackermann), Emigrantenliteratur (Biondi), Migrantenliteratur (Schierlob), Letteratura de-centrata (Sanna), Literatur in der Fremde (Chiellino), Eine nicht nur deutsche Literatur (Weinrich) o Literatur nationaler Minderheiten (Reeg), alusivas a la situación social de la persona del sujeto creador antes que a las condiciones estéticas de la obra.

El ánimo integrador implícito en la protección de la situación minoritaria del escritor inmigrante en estas primeras denominaciones permite rastrear en ellas, como una suerte de huella dendrocronológica, la evolución de este pensamiento en estos años iniciales. El esfuerzo por hacer visible ante la sociedad una realidad nueva a través de su verbalización en forma de denomina- ción literaria queda así patente en ellas. Sin embargo, esta forma de «sociologización de la literatura» (Stratthaus, 2005) trae consigo un efecto secundario: la promoción del aspecto espacialista del multiculturalismo, el de la consideración del canon de autores hijos de la inmigración como un segmento aparte, abonando así la Bereicherungsthese o la doctrina del Nebeneinander, próximas a una ghettización o aislamiento estanco de los nuevos espacios culturales que perpetúa las diferencias por medio del separatismo ético (Teraoka, 1996: 554) de los Ethnoblasen o de los Bindestrich-Deutsche (Zaimoglu, 1997).

La superación de este momento crisis viene de la mano de las propuestas teóricas próximas a la definición de la interculturalidad literaria desde mediados de los años noventa. La literatura intercultural es un espacio sin espacios, desterritorializado, que pone su acento en el camino antes que en el destino y que concibe el cambio como esencia de las relaciones culturales. La legión de nuevos teóricos de la interculturalidad literaria, como Carmine Chiellino (Universidad de Augsburg), Mustafa Al-Slaiman (Universidad de Mainz) o Aglaia Blioumi (Universidad de Atenas) reflejan en sus escritos y en la condición de parte de sus autores un nuevo estadio en la consideración de la inmigración en Alemania y, consecuentemente, en el signo de los estudios sobre contacto cultural.

La consolidación de los grupos de inmigración de segunda generación o con Migrationshintergrund genera una nueva identidad, la expresada literariamente por los Nichtdeutsche, die Deutsch schreiben (J.A. Oliver) o por la Literatur mit doppelter Staatsbürgerschaft (Blioumi) que reclama una nueva perspectiva que incluya en igualdad de condiciones esta doble identidad intercultural. Frente a la monoculturalidad imperante -también latente en el multiculturalismo del melting pot- emerge una nueva forma de biculturalidad que presupone esta condición en sus distintas dimensiones: en la condición del autor, de la literatura y de la crítica de doble nacionalidad. La nacionalidad única implícita en los modelos de categorización monoculturales es de este modo superada en favor de un nuevo modelo supranacional que deja atrás la reducción étnica nacida de la dialéctica entre mayorías y minorías culturales (Blioumi, 2003: 248).

En el sentido de Blioumi, el modelo de interculturalidad se aplicaría delimitando un segmento de la producción literaria, una minoría discriminada positivamente formada por un elenco de autores con una aportación sociológica determinada, temas, es- 
tilo y topografía de origen propios. Un modelo espacialista, en cualquier caso, que no perturba la dinámica de canonización literaria y que, fijando sus límites de admisión en la tercera generación de inmigrantes, perpetúa una forma distinta de monoculturalidad (Chiellino, 2000. 62).

No han faltado voces en estos años que han clamado contra esta oportunidad perdida de salvar las pesadas y camaleónicas barreras impuestas por el colonialismo cultural, fagocitador, universalista y eurocentrista, que aprovecha los eufemismos aplicados a nuevos territorios marginales, ya sean de literatura del emigrante, del asilado o del repatriado, para disponerles un espacio reservado en las categorizaciones críticas e histórico literarias. Primero Adelson (1991), luego Teraoka (1996) y recientemente Köstlin (2000) y Stratthaus (2006) han insistido en esta idea, que revela la incapacidad de esta visión de la interculturalidad para imprimir a los estudios literarios un carácter más dinámico y transversal; y la posibilidad de entenderla como categoría abierta y un modo de mirar antes que como una lista alternativa y cerrada de autores, como un modo de existir antes que como una oposición irresoluble entre origen y destino (Köstlin, 2000: 377).

Es justamente esta nueva coordenada introducida por esta crítica la que parece dar vivacidad a las últimas aportaciones sobre literatura intercultural. En ella, la disociación de la cultura del espacio y del territorio, la comprensión de la migración como un fenómeno histórico y natural, la aceptación del pasado cultural del recién llegado y de la necesaria tensión generada con su nuevo presente, acerca una definición de la literatura intercultural a lo que el teólogo Raimon Pannikar propusiera bajo el término interfecundación cultural, de acuerdo con la cual en la mirada a la diversidad de culturas prima la consideración de cada una de ellas como realidades inconmensurables.

Esta última apreciación pone a la interculturalidad en relación directa con una forma de entender el contacto cultural sensible a la permeabilidad entre los distintos sistemas, y en concreto, con la noción de transculturalidad inaugurada por el etnógrafo cubano Fernando Ortiz en los años cuarenta, refundada por Homi K. Bhabha en los años noventa y desarrollada en Alemania por Wolfgang Welsch (2000). La aportación en torno a esta noción en los últimos años ha sido prolífica, y supera la visión unicista para explicar, en su lugar, el contacto cultural como una relación total, alineando junto al parámetro de la lengua y la nación, el de la experiencia cultural de los individuos en materia de raza, género, o clase. Este enfoque que hace de la disciplina un observatorio cultural, permite detectar los esquemas culturalistas que laten tras la descripción de fenómenos, explorando una rica gama de categorías de análisis.

La transculturalidad mantiene parentesco semántico con otros términos afines como mestizaje, sincretismo, criollización o hibridismo. Justamente el hibridismo ocupa un lugar central en esta teoría, estudiado años atrás por teóricos como Breinig (2000), Lösch (2002), Mill, Allolio-Näcke, Kalscheuer y Manzeschke (2005), así como, más recientemente, por McPherson (2007), Schrader-Kniffki y Sandten (2007) y Saal (2007). Definido por Bhabha como el lugar de la diferencia exento de jerarquía, permite analizar la diferencia cultural -la transdiferencia- antes que como un juicio previo, como el resultado de la circunstancia de su expresión, intentando escrutar las posibilidades de explicación de por qué cambian los significados y las atribuciones de esas diferencias. De este modo se erige en una herramienta útil y actual para describir las identidades literarias y las relaciones de poder.

El progreso de la noción de interculturalidad describe, en definitiva, un recorrido paralelo al propio de la migración en la europa globalizada. Un proceso que registra distintas etapas en las que se definen el tempo y los modos, a la vez que la descripción de su correspondiente expresión literaria da cuenta de una observación cada vez más matizada de distintas fórmulas de contacto cultural: de una multiculturalidad aculturalista a una transculturalidad que contempla en su enfoque sistemas permeables, al tiempo que es sensible a la expresión de distintas y cambiantes cosmovisiones vivenciales en contacto.

\section{Comunicación intercultural ${ }^{2}$}

Hablar de interculturalidad en el campo de la comunicación significa sobre todo abordar el terreno de la comunicación intercultural muy debatido en los últimos años. Con este concepto se entienden fundamentalmente aquellas situaciones en las que dos individuos procedentes de un contexto cultural diferente se comunican mediante la lengua o expresiones no verbales. Gracias en gran medida a la facilidad de las comunicaciones interpersonales y geográficas entre los diferentes países y culturas, al intercambio económico y a los movimientos migratorios, y debido a los problemas en los procesos de comunicación derivados de éstos, ha surgido la necesidad de estudiar un «nuevo mundo» con una perspectiva interdisciplinar y práctica; interdisciplinar porque resulta muy útil combinar varias disciplinas como la lingüística, la psicología, las ciencias económicas, la pedagogía, la antropología y la Kulturwissenschaft, y esta nueva óptica se convierte de esta manera en una especie de soft skill, una habilidad que afecta a varias disciplinas de las que al mismo tiempo es independiente (Erll/Gymnich 2007: 7); y práctica porque está estrechamente relacionada con términos como competencias y habilidades interculturales o mediación intercultural, que a su vez parecen establecer vínculos más directos con problemas diarios de integración o de (in) comprensión entre diferentes realidades culturales. Basta en este sentido echar un vistazo a los títulos de las obras publicadas que incluyen el término interculturalidad. Algunos de ellos rayan la frontera de los manuales de utilidad social que no se podrían calificar precisamente como teóricos o científicos. ${ }^{3}$

Tampoco los centros universitarios se han escapado del campo de estudio de la comunicación intercultural. En Alemania no son pocas las universidades que ofertan títulos de máster en Comunicación Intercultural bajo este nombre o similar (Erll/Gymnich 2007: 164-167). En el sistema universitario español sin embargo se puede detectar una casi absoluta au- 
sencia de investigación en este campo.

Sin embargo, antes de la mencionada explosión mediática de este nuevo término, surgido principalmente de las relaciones económicas entre pueblos de diferentes culturas, se han realizado estudios teóricos que han abordado aspectos generales sobre el concepto de cultura por un lado, y elementos específicos sobre aspectos de tipo lingüístico por otro, en ambos casos procedentes de Estados Unidos. Quizás los primeros han tenido mayor repercusión, y de manera especial los estudios de Edward Hall (1951, 1966, 1976), que se pueden encuadrar dentro de la sociología, la antropología y la psicología, con el objeto de describir conceptos como la proxémica, policromía o comunicación intercultural, aportado por él mismo en 1959. Hall intenta superar algunos de los problemas interculturales entre los diferentes países mediante la observación y el análisis (como por ejemplo el concepto de esfera privada en diferentes culturas). Esta tendencia a resolver problemas prácticos viene dada básicamente por las circunstancias propiciadas por el final de la Segunda Guerra Mundial y especialmente con la fundación de organismos internacionales como la $\mathrm{ONU}$ con el objetivo de velar entre otras cuestiones por la defensa de la diversidad cultural.

Entre los segundos tipos de estudios más específicos -más concretamente en el campo de la lingüística- hay que resaltar sin duda los primeros estudios contrastivos de los años $20 \mathrm{y}$ 30 , pero fundamentalmente los trabajos realizados por Robert

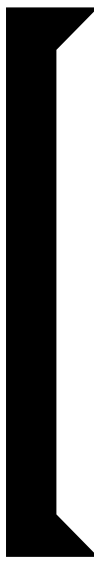

El progreso de la noción de interculturalidad describe, en definitiva, un recorrido paralelo al propio de la migración en la EuLado (1957) en su obra teóriropa globalizada. ca sobre la lingüística contrastiva Linguistics across cultures con un enfoque fundamentalmente lingüístico en el que llega a la conclusión de que expresar diferentes realidades en diferentes lenguas requiere una confrontación más amplia de lo que a priori ofrece la lingüística del momento. La lengua no es un conjunto cerrado, sino una habilidad constantemente en evolución influenciada por el entorno, que repercute no sólo en cuestiones de semántica y pragmática, sino también en cuestiones como la enseñanza de segundas lenguas. No obstante, siempre se le ha achacado la ausencia de un modelo teórico. ${ }^{4}$ También hay que mencionar obras de carácter introductorio que parten desde una base lingüística, especialmente del análisis del discurso, ámbito que permite confrontar y abordar aspectos como estrategias de comprensión, modelos de comunicación o malentendidos bajo un prisma discursivo no sólo entre individuos de diferente cultu- ra, sino incluso de la misma (Grein/Weigand 2007, Erll/Gymnich 2007, Heringer 2004 entre otros).

En la actualidad los modelos teóricos más relevantes basados en el proceso comunicativo se preguntan básicamente por la naturaleza y los momentos de la comunicación intercultural dentro y fuera del discurso. En los siguientes tres modelos ${ }^{5}$ se observa una aportación principalmente desde perspectivas integradoras, aunque con métodos diferentes (Rodrigo 1999: 164).

La Teoría de la Reducción de la Ansiedad y de la Incertidumbre, desarrollada por Gudykunst (1983) y aún sin acabar, pues como afirma el propio autor, se encuentra en constante evolución, pone el acento sobre aspectos relacionados con el proceso comunicativo, tanto verbales, -qué información se transmite-, como no verbales, -qué tipo de gestos o circunstancias acompañan y rodean al contenido semántico, es decir, cómo y en qué condiciones se transmite el mensaje-. Gudykunst explica el proceso comunicativo a partir de conceptos como stranger (el individuo que representa lo desconocido y el causante de mecanismos de ansiedad e incertidumbre), anxiety (respuesta emocional a lo desconocido) o uncertainty (no se puede predecir la conducta del forastero). La toma de conciencia de estas circunstancias y de las expectativas de los hablantes de diferente cultura contribuye a la superación de los problemas que pueden surgir de una situación comunicativa intercultural. Se trata sin duda de un modelo teórico complejo que involucra más bien las competencias afectivas que las cognitivas y pragmático-comunicativas.

Desde una perspectiva más global enfoca el problema de la comunicación intercultural la Teoría de la Construcción de la Tercera Cultura. L. Casmir (1993), apoyándose en la Escuela de Palo Alto -entre los que se encuentra P. Watzlawick- afronta la comunicación interpersonal e intercultural más bien como un punto de encuentro, donde "no se trata de vencer, sino de convencer» (Rodrigo 1999: 207). La tercera cultura, fruto del «roce» entre dos culturas diferentes representa un lugar de encuentro donde resolver los procesos comunicativos conflictivos; es una especie de subcultura que reconoce la diversidad de las culturas en contacto con un carácter analítico.

Al contrario que Casmir, y más bien cercana a la Teoría de la Gestión de la Ansiedad, Kim propone (1995) la Teoría de la Adaptación Transcultural como modelo para la comunicación intercultural. Las bases se hunden en el esfuerzo de integración que debe hacer el individuo forastero en la cultura de destino. Ello supone dos fases para el forastero, una primera de deculturación, en la que olvida algunas de las costumbres de la cultura nativa, y al mismo tiempo una fase de aculturación, que conlleva el aprendizaje de hábitos de la cultura de acogida.

En definitiva, todas estas teorías están basadas en el empirismo y en este sentido recogen algunas de las formas reales de la 
comunicación intercultural sobre todo en la experiencia personal. Con seguridad permiten reflexionar de una forma u otra sobre realidades más complejas. Este tipo de enfoques resultan imprescindibles en los estudios universitarios no sólo en Alemania, sino también en España, especialmente considerando el hecho de que hoy ya un $10 \%$ de la población procede de realidades y culturas diferentes y en consecuencia aumenta la necesidad no sólo de integrar elementos heterogéneos, sino de comprender desde la cercanía de la convivencia experiencias y bagajes culturales que hasta hace poco tiempo parecían infinitamente lejanos

\section{Bibliografía}

Adelson, L. A. (1991) «Migrantenliteratur oder deutsche Literatur? TORKANs Tufan: Brief an einen islamischen Bruder», en: Lutzeler, P.M. (ed.), Spätmoderne und Postmoderne. Beiträge zur deutschsprachigen Gegenwartsliteratur, Frankfurt am Main: Fischer, 67-81.

Bhabha, Homi K. (2000): Die Verortung der Kultur, Tübingen: Stauffenburg. Original: (1994): The Location of Culture, London: Routledge.

Blioumi, A. (2001): «Gegen Kulturdifferenzen? Der Begriff des ,Universalbildes' als analytische Kategorie für die Literaturwissenschaft», Neobelicon, 28, 89-99.

Blioumi, A. (2003): «Amerikanischer Multikulturalismus und deutsche Interkulturalität. Zur Rezeption der Migrationsliteratur in den USA und in Deutschland", Neohelicon, 30, 243-249.

Blioumi, A. (2004): «Kulturaustausch, Interkulturalität und Interdisziplinarität. Beispiele aus der deutschsprachigen Migrationsliteratur", Neohelicon, 31, 43-59.

Blioumi, A. (2006): "Migrationsliteratur oder das Monster von Loch-Ness», en: Blioumi, A. Transkulturelle Metamorphosen. Deutschsprachige Migrationsliteratur im Ausland am Beispiel Griechenland. Würzburg: Königshausen \& Neumann, 26-29.

Casmir, F. L. (1993): «Third-Culture Buildings: A Paradigm Shift for International and Intercultural Communication», en: Deetz, S.A. Communication Yearbook/16, London: Sage, 407-428.

Chiellino, C. (2000) (ed.): Interkulturelle Literatur in Deutschland. Ein Handbuch, Stuttgart: Metzler, 1-535.

Chiellino, C. (2000): «Einleitung: Eine Literatur des Konsenses und der Autonomie - Für eine Topographie der Stimmen", en: Chiellino, C. (ed.), Interkulturelle Literatur in Deutschland. Ein Handbuch, Stuttgart: Metzler, 51-63.

Chiellino, C. (2000): «Interkulturalität und Literaturwissenschaft», en: Chiellino, C. (ed.), Interkulturelle Literatur in Deutschland. Ein Handbuch, Stuttgart: Metzler, 387-399.

Erll, A./Gymnich, M. (2007): Interkulturelle Kompetenzen. Erfolgreich kommunizieren zwischen den Kulturen, Stuttgart: Klett.

Grein, M. / Weigand, E. (2007): Dialog and Culture, Amsterdam: John Benjamin.

Gudykunst, W.B. (1983): Intercultural Communication Theory. Current perspectives, London: Sage.

Gudykunst, W.B. (2005): Theorizing about Intercultural Communication, Thousand Oaks, CA: Sage.

Hall, E. T. (1951): The silent language, Garden City, NJ: Anchor Books/Doubleday.
Hall, E. T. (1966): The hidden Dimension, Garden City, NJ: Anchor Books/Doubleday.

Hall, E. T. (1976): Beyond Culture, Garden City, NJ: Anchor Books/Doubleday.

Herbrand, F. (2002): Fit für fremde Kulturen. Interkulturelles Training für Führungskräfte, Bern: Haupt.

Heringer, H.J. (2004): Interkulturelle Kommunikation, Tübingen: Francke.

Kahle, Christiane (2005): «Interview mit Feridun Zaimoglu: 'Der Islam ist eine Modewelle'», [en línea] [consulta: octubre de 2007], disponible en: http://www.tagesschau.de/inland/meldung203352.html.

Kim, Y. Y. (1995): "Cross-Cultural Adaptation. An Integrative Theory», en Wisemann, R. L. (ed.), Interculltural Communication Theory, London: Sage, 170-193.

Klappenbach, D. (2006): Mediative Kommunikation, Paderborn: Junfermann.

Köstlin, K. (2000): «Kulturen im Prozeß der Migration und die Kultur der Migrationen", en: Chiellino, C. (ed.), Interkulturelle Literatur in Deutschland. Ein Handbuch, Stuttgart: Metzler, 365-387.

Kumbier, D. / Schulz von Thun, F. (2006): Interkulturelle Kommunikation: Methoden, Modelle, Beispiele, Hambug: Rowohlt.

Lado, R. (1957): Linguistics across cultures, Michigan: University of Michigan Press.

Losche, H. (2005): Interkulturelle Kommunikation. Sammlung praktischer Spiele und Übungen, Augsburg: Ziel.

Mayer, C-H. (2006): Trainingshandbuch Interkulturelle Mediation und Konfliktlösung. Didaktische Materialien zum Kompetenzerwerb, Münster: Waxmann.

Mc Pherson, A. (2007): «Trans-Formationen: Aufgaben und Grenzen transkultureller Analyse-Ansätze», en: Sandten, C.; SchraderKniffki;Starck, K. Transkulturelle Begegnungen, Trier: Wissenschaftlicher Verlag Trier, 17-34.

Pigem, J. (2001): «Interculturalidad, pluralismo radical y armonía invisible», Ilu, Revista de Ciencias de las Religiones, anejo 6 [Homenaje a Raimon Pannikar: Sam_dh_nam], 117-131.

Raddatz, F. (1994): «In mir zwei Welten», en Die Zeit, 26, 45-46.

Rein, K. (1983): Einfübrung in die kontrastive Linguistik, Darmstadt: Wissenschaftliche Buchgesellschaft.

Rodrigo, M. (1999): Comunicación intercultural, Barcelona: Anthropos.

Saal, B. (2007): «Kultur in Bewegung. Zur Begrifflichkeit von Transkulturalität», en: Mae, M.; Saal, B. (eds.), Transkulturelle Genderforschung. Ein Studienbuch zum Verhältnis von Kultur und Geschlecht, Wiesbaden: VS Verlag für Sozialwissenschaften, 21-36.
Schrader-Kniffki, M.; Sandten, C. (2007): «Einleitung: Transkulturelle Diskurse und transkulturelle Ausdrucksformen", en: Sandten, C.; Schrader-Kniffki; Starck, K. Transkulturelle Begegnungen, Trier: Wissenschaftlicher Verlag Trier, 1-14.

Stratthaus, B. (2006) Was heißt «interkulturelle Literatur»? [tesis doctoral], 21.2.05, Universität Duisburg-Essen, 1-264.

Teraoka, A. A. (1996): «Deutsche Kultur, Multikultur: Für eine Germanistik im multikulturellen Sinn», Zeitschrift für Germanistik, VI, 3, 545-560.

Tibi, B. (1998) Europa ohne Identität? Die Krise der multikulturellen Gesellschaft. Múnich: Bertelsmann, 1-379.

Weinrich, H. (1984): "Gastarbeiterliteratur in der Bundesrepublik Deutschland", Zeitschrift für Literaturwissenschaft und Linguistik, $14 / 56,12-22$.

Welsch, W. (2000): «Transkulturalität. Zwischen Globalisiserung und Partikularisierung\}, en: Alois Wierlacher et. al. Jabrbuch Deutsch als Fremdsprache. Intercultural German Studies, 26, 327-351.

\section{Notas}

1 El presente artículo se compone de dos partes diferenciadas. La primera parte, de la que es autor Víctor Manuel Borrero Zapata y que aborda la literatura intercultural, se inscribe en el Proyecto I+D+I «Literatura e identidad cultural. La interpretación del pasado en la narrativa alemana a partir de 1945» (P.N. 2006; HUM2006-03572) financiado por el Ministerio de Educación y Ciencia, y recoge parte de la información elaborada en el capítulo «Canon e identidad cultural» que se publicará próximamente dentro de la monografía Literatura e identidad cultural. La interpretación del pasado en la narrativa alemana a partir de 1945, en el marco del mismo proyecto. La segunda parte ha sido elaborada por Javier Martos Ramos y trata la comunicación intercultural.

2 Quisiera agradecer a Leonarda Trapassi por los comentarios acerca de las teorías de la comunicación intercultural.

3 En el campo de la investigación intercultural alemana,se encuentran Losche (2005), Herbrand (2002), Kumbier (2006), Mayer (2006) o Klappenbach (2006) entre muchos otros.

4 La cuestión es cómo se expresa una acción en una lengua y no cómo se traduce, lo que en opinión de Coseriu confería gran fragilidad teórica al contraste de lenguas (Rein 1983).

5 Gudykunst (2005) habla de seis tipos de teorías, pero las tres que se describen son las más relevantes para el proceso comunicativo. 


\section{Eine Reise zum Mittelpunkt...}


\section{...müssen Sie nicht unternehmen.}

Wir schicken Ihnen einfach ein Probeexemplar zu.

$\begin{array}{lll}\text { difusión } & \begin{array}{l}\text { Difusión, Centro de Investigación } \\ \text { y Publicaciones de Idiomas, S.L. } \\ \text { c/ Trafalgar, 10, entlo. 1a 08010 Barcelona }\end{array} \\ \begin{array}{l}\text { Tel.: (+34) } 932680300 \\ \text { Fax: (+34) } 933103340 \\ \text { www.difusion.com }\end{array}\end{array}$

